$\xi=-1$

\title{
Integration of DERs for Optimal Sharing of Power in Grid Connected Mode as Well as Islanded Mode
}

\author{
L. Bangar Raju ${ }^{1}$, K.Subba Rao ${ }^{2}$ \\ ${ }^{1}$ Research scholar, ${ }^{2}$ Professor, ${ }^{1,2}$ Department of Electrical \& Electronic Engineering \\ ${ }^{1,2}$ Koneru Lakshmaiah Education Foundation, Vaddeswaram, Guntur, Andhra Pradesh, INDIA \\ *Corresponding AuthorEmail: ${ }^{1}$ lsmlbr@yahoo.in , ${ }^{2} l k s b \_a \_e e e @ k l c e . a c . i n$
}

\begin{abstract}
The Microgrid consists of distributed energy resources, DERs(distributed generators - DGs) and distributed storages - DSs), loads, PCC, energy management controllers etc., which are required for effective, efficient, reliable and seamless operation of microgrid in both grid connected mode and islanded mode. The heart of the microgrid is the integration part of the DERs with energy management controllers. Out of the methods available, hierarchical method of integrating DERs is selected in this paper. The method is based on the principle of hybrid particle swarm optimization (PSO) which takes into account of genetic algorithm (GA) and PSO. In this paper, the best part of particle to particle information sharing in GA is combined with fast convergence option in PSO, to get the optimum solution with minimum no of iterations. Integration of DERs is nothing but energy management in supply side management (SSM) as well as demand side management (DSM). DSM consists of load shedding or load shifting to an alternate feeder. The primary energy controller MC (microsource controller) controls voltage and frequency through droop control inverters. The secondary controller (MGCC) monitors the flow of active and reactive power in the microgrid and communicates to the DMS (distribution management system). The tertiary controller, DMS takes care of overall control of the network like export, import, islanding, economical aspects of the DERs operation in the microgrid. The energy management module (MGCC) must also contain protection co-ordination module (PCM) along with energy management module (EMM), for relay coordination as microgrid consists of two operating modes viz., grid connected mode and islanded mode. The fault currents substantially reduces to smaller values in islanded mode as DERs inertias are low. For this reason the PCM must have two settings and auto changeover of settings are required from one mode to another by PCM module to suit islanding and grid modes.
\end{abstract}

Keywords: DOE-department of energy; US-united states; EU - Europe; LV-low voltage; DERs-distributed energy resources; DG-distributed generation,; DSsdistributed storage systems; PV-photo voltaic; PSO - particle swarm optimization; GA - genetic algorithm; ESS - energy storage systems; DLs - demand loads,;PCC - point of common coupling; SSM - supply side management; DSM - demand side management; LC- load controller;LS - load shedding / load shifting; MGCC / CC - microgrid central controller; EMM - energy management module; PCM - protection co-ordination module; CHP - combined heat and power; $M P P T$ - maximum power point tracking; OPL - Optimal power flow;

\section{Introduction}

As per DOE, US, Microgrid is a subset of power grid, defined as that which acts as a single controllable entity with all its DERs and loads within the electrical boundaries and can connect and disconnect with the Macrogrid or utility grid to operate in grid or islanded mode. EU also defines Microgrid in the same way but defines as a LV ac active distribution network [1]

Whatever it is, the operation of micro-sources must be beneficial to the community with overall network performance, if managed and co-ordinated efficiently. There are different types of Microgrids, viz., community, remote, military, commercial or industrial Microgrids. The microgrid has to fulfill the following six functions, 1. Frequency and voltage regulation

2. Spinning reserve
3. Standalone operation

4. Seamless operation from grid mode to islanded mode

5. Peak shaving

6. Load shedding / load shifting

For these functions to be undertaken by microgrid, there are four methods of DERs integrating methods are available, which are called energy management systems. They are centralized, decentralized, hierarchical and local control methods. In a centralized control a big amount of data information transfer between the units is required. But in a decentralized control, each unit is controlled by its own controller without knowing the status of other units. Both the schemes are not feasible. Hence hierarchical control scheme consisting primary, secondary and tertiary control levels is practical for a better solution. The hierarchical control can also be called as distributed and are suitable for microgrid with no of DERs. The isolated mode is not suitable for microgrid with more 
DERs. The primary control level in hierarchical control is the most fastest (from milliseconds to seconds), which controls voltage, frequency, sharing of active and reactive power, mitigate circulating currents in DERs(DGs+DSs), to provide voltage and current loops for secondary controller. The secondary controller controls the deviations of frequency and voltage given by primary controller. This is a slower controller (milli seconds to seconds) and thus maintains power quality for voltage balance at critical buses. Tertiary control level is the slowest (sampling between seconds to minutes) and takes care of the economical operation for the power flow between grid and Microgrid, which will be useful for "Cluster of Microgrids" operation. During this stage one of the Microgrids, is to be selected as slack or master and the rest as PV and load buses as per the algorithm. In this case the control should be based on real time or high sampling rate. A new hybrid genetic algorithm (GA) cum particle swarm optimization(PSO) technique is adopted for DERs integration, control, protection, operation and restoration. The main components of Microgrid are mini-hydro, solar cell, wind energy, fuel cell and energy storage system. These are integrated in Microgrid for generation, energy storage, and loads, that normally operate connected to a main grid (utility grid). Microgrid can operate in two modes: one is grid-connected and the other is stand-alone or isolated mode. The main benefit of Microgrid is the one which can operate in islanded mode or utility grid connected mode or both. The Microgrid in standalone mode can function autonomously. Generation and loads in a Microgrid are generally interconnected at low voltage. But one issue related to Microgrid is that, operator should be very vigilant because number of micro-sources are connected to Microgrid. In the past, in utility grid, the power flow is in one direction only. Then they used to be called as "Passive Distribution Networks. But latest Microgrids with distributed energy resources such as fuel cells, wind, solar, energy storages and controllable loads, the power flow direction is in two directions and hence they are called "Active Distribution Networks. These multiple different electric power supply generation units have the ability to supply power to loads, even when the Microgrid is isolated from utility grid and provide "reliable electric power. Even when the Microgrid is in grid mode, peak shaving is possible with DERs integration. The most important task in a microgrid is the integration of DERs and DLs for optimum load sharing between DGs and ESS.[2].

\section{Methodology for DERs Integration}

The following are required for implementation in Microgrid system for integration,

- First we need to select electric power supply system such as Distributed Energy Resources (DERs) according to requirement and availability of input sources

- Select Energy Storage System (ESS) and Energy Management System(EMS) [8]

- Then we need to integrate all these resources (DERs) in Microgrid such as Distributed Energy Resources(DGs + ESSs)[10][13][14].

- Then implement Energy control and Energy Management System [9]

\subsection{Microgrid Architecture}

Microgrid architecture is shown in Fig.1with all its DERs.

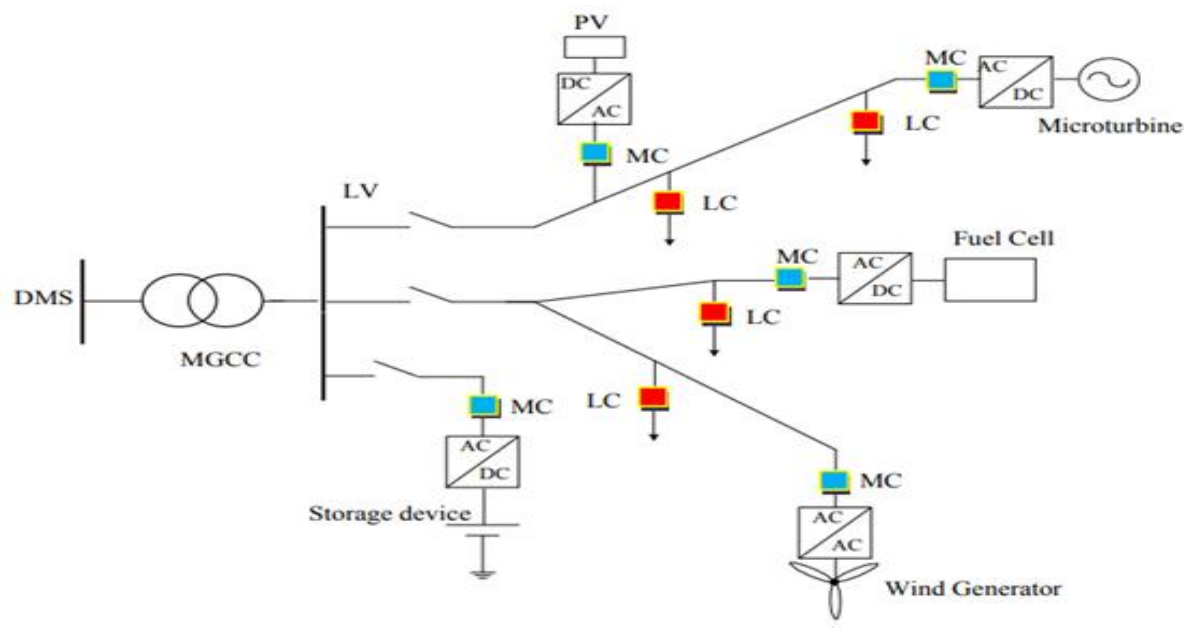

Fig. 1: Microgrid Structure

$\mathrm{PV}$ - photo voltaic - output is DC

Wind Generator - wind turbine - output is AC

LV - low voltage

DMS - distribution management system

MGCC - microgrid central controller

$\mathrm{MC}-$ microsource controller

LC - load controller

Fuel Cell - output is DC

Storage Device - can be batteries, flywheel, capacitors etc.,

Renewables are solar, wind and CHP

\subsection{Types of DERS and characteristics}

\section{Types of DERs}

Distributed energy resources include distributed generations like solar, wind, bio-mass, fuel cell, CHP, diesel generator, IC engine, micro-turbines etc. and distributed storages like batteries, flywheel, super capacitors etc. 


\section{DERs characteristics}

PV - The output depends on radiation levels and interface is through power electronic inverter (DC-DC-AC) and control is MPPT \& DC link voltage controls

$(+\mathrm{P}, \pm \mathrm{Q})$

Wind Generator - The output depends on wind velocity. The interface is through power electronic

inverter (AC-DC-AC) and control is

MPPT, pitch \& torque controls $(+\mathrm{P}, \pm \mathrm{Q})$

Microturbine-The output depends on geographical

location and the interface is through

Synchronous or induction generator. The power flow control is $(+\mathrm{P}$, $\pm \mathrm{Q})$

Fuel Cell - The output depends on source and the interface

is through inverter (DC-DC-AC)

\subsection{DERs capacities Selected for Microgrid}

The load profiles have been taken from a small residential area which comes to $90.05 \mathrm{KW}$ peak and daily energy consumption is $790 \mathrm{KWh} /$ day.

The resource input data is collected over a period of one year from the above small residential area, to obtain optimal design values. The average wind speed and solar radiation are calculated, to compute optimal solar power, wind power design values. Based on the following mathematical model. The results are given below after the formulae are derived..

\subsubsection{PV Generator}

Photovoltaic cells are connected in series or parallel to form modules and in turn form arrays. The power generated by solar panels depends on ambient temperature and solar irradiation. There are two options for calculating power output from solar plant[2]

\subsubsection{Without MPPT}

For no tracking system the output of PV generator is considered fixed by battery voltage so

VbusDC = Vnpanel .Npanel_series

Where Vbus dc is the dc bus voltage, Vn_panel is the nominal voltage of the PV panel and Npanels_series is the number of panels in series.

In such case power supplied by the photovoltaic generator is calculated as $\mathrm{P}$,

P = Isc G. Vnpanel .Npanel_seriesNpanel_parallel/LF

Where Isc is the short circuit current of panel, $\mathrm{G}$ is the irradiance on the surface of the panels in KW/m2 and L.F is

the loss factor. In this case output power practically does not depend on ambient temperature.

\subsubsection{With MPPT}

The power generated by the PV generator considering the effect of temperature is calculated as $\mathrm{P}, \mathrm{P}=$

(Pn.G(1+Ct/100.(Tc25)Npanels.seriesNpanels.paralle/FS

$\mathrm{Tc}=\mathrm{Tamb}+\mathrm{G} \cdot(\mathrm{TONC}-20) / 100$
Where Pn is the nominal power (Wp) of PV panel, Tc is the internal cell temperature, $\mathrm{Ct}$ is the maximum power, temperature coefficient, Tamb is the ambient temperature and F.S is the safety factor.

In a small PV system with battery storage number of panels in series is calculated,

Npanels_series =VbusDC/ Vn_panel

For high power the number of panels in series are calculated by,

Npanels_series =VbusDC / Vmax_p_panel

Where Vmax_p_panel is the voltage of the maximum power of the PV panels, Npanels_parallel< Peak power of generator. Npanel_series/

Vn_panel

\subsubsection{Wind Turbine}

The power generated from wind turbine is given by [4]

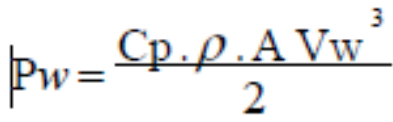

Where $\mathrm{Cp}$ is the power coefficient, $\mathrm{Vw}$ is the wind speed, $\mathrm{A}$ is the area of swept by the rotor and $\rho$ is the air density.

Amount of aerodynamic torque $\mathrm{Tw}$ is given by,

$\mathrm{Tw}=\mathrm{Pw} / \mathrm{Ww}$

Where $\mathrm{Pw}$ is the power extracted from the wind and $\mathrm{Ww}$ is the turbine rotor speed.

Air density and atmospheric pressure are given by,

$\rho=$ Pa.M/1000.R.T

$P a=P o \cdot\left(1-\frac{L \cdot H}{T o}\right)^{\frac{g M}{R L}}$

\section{Battery System}

Batteries play an important role as they are back up for the solar and wind plants. through inverters, to supply to loads for reliable power supply. The battery capacity is determined

from the load consumption pattern of the day.

The battery storage capacity is expressed in Cwh, which is equal to,

$$
C w h=\frac{\left(E_{D L} A D\right)}{\left(\eta_{\mathrm{BDI}} \cdot \eta_{\mathrm{B}} \cdot \mathrm{DOD}\right)}
$$

Where $\mathrm{AD}$ is number of autonomy days, $\mathrm{DOD}$ is the allowable depth of discharge of battery, $\eta \mathrm{BDI}$ is the bidirectional inverter efficiency and $\eta \mathrm{B}$ is the battery efficiency.

\subsubsection{Charge Regulator}

Charge regulator controls the charge flow from battery to load and solar and wind panels to battery. The role of charge regulator is very important as output from solar and wind plants are unpredictable. The charge regulator protects battery, solar and wind panels againest overcharging, fast discharging and blocks reverse charge and maintains natural energy. 


\subsubsection{Bidirectional Converter}

Power output is connected to load via bidirectional converters. Converter rating is selected based on. the peak demand of load and battery charging. These converters have to take care of energy management of DERs.

\subsubsection{Ders Design Values}

The final DERs design values from the formulae

of the above are given below. The optimal values of global solar radiation and wind speed as a sensitivity variable is given below.

Solar radiation $-3.22 \mathrm{~m} / \mathrm{s}$

Wind speed $-5.79 \mathrm{kwh} / \mathrm{m} 2 / \mathrm{d}$

PV array - 35Kw, 220Wp, $12 \mathrm{~V}$ polycrystalline modules

(panel)

Wind turbine $-50 \mathrm{kw}$

Fuel Cell - $20 \mathrm{kw}$

Battery Bank - 4500kwh

Battery Charger - 177A

Inverter / Rectifier - 150kva

Load connected - $90.05 \mathrm{KW}$ peak,

Daily energy consumption - $781.69 \mathrm{KWh} /$ day

These design values have been tested in hybrid software, for sizing and integration of DERs.. The sensitivity analysis will be useful for accessing the uncertainties of PV and Wind energies to find the most suitable solution of the hybrid system.

\section{Hybrid Optimization Method}

The objective of the hybrid optimization method is to control parameters like load flow, generator bus voltages, load bus voltages, active and reactive power flows[12]. To get away with these problems, the methodology adopted is given below.

\subsection{Optimization Hybrid Algorithm}

The hybrid system combines GA and PSO methods to form a hybrid algorithm. Due to this combination the off-springs can keep diversity and PSO can keep balance between global search and local search. By doing so the entire search ability of the algorithm is improved.

The hybrid system maintains the integration of GA and PSO for the entire run, which consists of genetic algorithm, combined with PSO and the procedure to adopt the algorithm is given below

1: Randomly initialize the population of $\mathrm{P}$ individuals within the variable constraint range.

2: Calculate the fitness of the population from the fitness function in the ascending order..

3: The top $\mathrm{N}$ individuals are selected as the elites and reproduce them directly to the next generation

4: The $\mathrm{S}$ individuals followed are evolved with PSO and their best positions are updated.

5: The bottom individuals are evolved with hybrid GA plus PSO and produce P-S-N offspring.

6: Combine the three parts as the new generation and calculate the fitness of the population. Choose the best position among all the individuals obtained so for the global best.

7: Repeat steps 3-6 until a stopping criterion, such as a sufficiently good solution being discovered or a maximum number of generations being completed, is satisfied. The best scoring individual in the population is taken as the final answer.

\subsection{Basis for Different Optimization Techniques}

\section{Improved Genetic Algorithm}

GA uses the real variables and thus is free from binary encoding and decoding. It takes less memory space and works faster than binary GA. Some practical schemes to improve GA performance are adopted. According to the optimal results, we can conclude that these measures are effective and helpful in improving convergence property and accuracy[17].

\section{Nonlinear Ranking Selection}

Ranking methods only require the evaluation function to map the solutions to a partially ordered set. All individuals in a population are ranked from best to worst based on their fitness values. It assigns the probability of an individual based on its rank ( $r$ ) and it is expressed as follows,

\section{$\{1$, for the best individual \\ $\{P$, for the worst individual \\ $P=$ the population size}

It can be seen that this selection probability doesn't use the absolute value information of fitness value so that it avoid the fitness value scale transformation and control the prematurity to some extent.

\section{Competition and Selection}

In natural biological evolution, two parents after crossover can produce several offspring, and the competition also exists among the offspring which are produced by the same parents. Motivate by this, phenomenon, we adopt competition and selection among several crossover offspring. Different from the conventional algorithm in which two parents only produce two offspring, the two parents, chromosomes as,

$a_{s}=\left[x_{1}^{s}, x_{2}^{s}, \ldots, x_{n}^{s}\right]$ and $a_{t}=\left[x_{1}^{t}, x_{2}^{t}, \ldots, x_{n}^{t}\right]$

in this algorithm will produce four chromosomes according to the following mechanisms,

$$
\begin{aligned}
& b_{1}=\left[b_{1}^{1}, b_{2}^{1}, \ldots, b_{n}^{1}\right]=\frac{a_{s}+a_{t}}{2} \\
& b_{2}=\left[b_{1}^{2}, b_{2}^{2}, \ldots, b_{n}^{2}\right]=a_{\max }(1-\omega)+\max \left(a_{s}, a_{t}\right) \omega \\
& b_{3}=\left[b_{1}^{3}, b_{2}^{3}, \ldots, b_{n}^{3}\right]=a_{\min }(1-\omega)+\min \left(a_{s}, a_{t}\right) \omega \\
& b_{4}=\left[b_{1}^{4}, b_{2}^{4}, \ldots, b_{n}^{4}\right]=\frac{\left(a_{\max }+a_{\min }\right)(1-\omega)+\left(a_{1}+a_{2}\right) \omega}{2} \\
& a_{\max }=\left[x_{1}^{\max }, x_{2}^{\max }, \ldots, x_{n}^{\max }\right] \\
& a_{\min }=\left\lfloor x_{1}^{\min }, x_{2}^{\operatorname{men}}, \ldots, x_{n}^{\min }\right]
\end{aligned}
$$

Where, we $[0,1]$ denotes the weight to be determined by users, $\max \left(a_{s}, a_{t}\right)$, denotes the vector with each element obtained by taking the maximum among the corresponding element of as and at . Among b1 to b4, the two with the largest fitness value are used as the offspring of the crossover operation. At the same time, (16) and (17) results in searching around the centre region of the domain, (18) and (19) can move to be near amax and amin respectively. Thus, the offspring generated by this operator, is better than that obtained by arithmetic crossover or heuristic crossover. 


\section{Mutation}

This is the unary operator responsible for the fine tuning capabilities of the system, so that it can escape from the trap of local optimum. It is defined as follows: For a parent $p$, if variable pk was selected at random for this mutation, the result is,

$$
\begin{aligned}
& \bar{P}=\left(P_{1}, \ldots, \overline{P_{k}}, \ldots, P_{n}\right) \\
& \overline{P_{k}}=\varepsilon \quad\left\{\max \left(P_{k}-\mu \frac{P_{k}-P_{k}}{2}, P_{k}^{\min }\right), \min \left(P_{k}+\right.\right. \\
& \left.\left.\mu \frac{P_{k}^{\max }-P_{k}^{\min }}{2}, P_{k}^{\max }\right)\right\}
\end{aligned}
$$

and $P_{k}^{\max }, P_{k}^{\min }$ are upper and lower bounds of $\mathrm{Pk}$ respectively, decreased with the increase of iterations.,

$\mu(\tau)=1-r^{[1-(\tau / T)]^{b}}$

where $\mathrm{r}$ is uniform random number in $[0,1], \mathrm{T}$ is the maximum number of iterations, $r$ is the current iteration number, and $b$ is the shape parameter. From (34), at the initial stage of evolution, for small value of $r, \mu(r) \approx 1$, the mutation domain is large in this case. However, in the later evolution, when approaches $\mathrm{T}, \mu(\mathrm{r}) \approx 0$, the mutation domain become small and search in the local domain.

\subsection{Particle Swarm Algorithm}

PSO was developed by James Kennedy and Russell Eberhart in 1995. Genetic algorithm (GA) in particular became popular through the work of John Holland in the early 1970s [17 ].The PSO conducts searches using a population of particles which correspond to individuals in GAs. The population of particles is

$$
\left\{\begin{array}{c}
p(r)=q^{\prime}(1-q)^{r-1} \\
q^{\prime}=\frac{q}{1-(1-q)^{p}}
\end{array}\right.
$$

So that,

$$
\sum_{r=1}^{p} p(r)=1
$$

where

$\mathrm{q}=$ the probability of selecting the best individual $=[0,1]$,

$\mathrm{r}=$ the rank of the individual $=$

randomly generated initially. Each particle represents a potential solution and has a position represented by a position vector $\overrightarrow{x_{2}}$. A swarm of particles moves through the problem space, with the moving velocity of each particle represented by a position vector, . $\overrightarrow{v_{l}}$.At each time step, a function $f_{i}$.representing a quality measure is calculated by using $\overrightarrow{x_{z}}$. as input. Each particle keeps track of its own best position, which is associated with the best fitness it has achieved so far in a vector $\vec{p}_{l}$. Furthermore, the best position among all the particles obtained so far in the population is kept track of as $\overrightarrow{p_{g}}$. At each time step $\boldsymbol{\tau}$, by using the individual best position $\overrightarrow{p_{\imath}}(\boldsymbol{\tau})$, and global best position, a new velocity for particle $\mathrm{i}$ is updated by

$\overrightarrow{v_{l}}(\tau+1)=\omega \overrightarrow{v_{l}}(\tau)+c_{1} \emptyset_{1}\left(\overrightarrow{p_{t}}(\tau)-\overrightarrow{x_{i}}(\tau)\right)+$ $c_{2} \emptyset_{2}\left(\overrightarrow{p_{g}}(\tau)-\overrightarrow{x_{i}}(\tau)\right)$

Where $\mathrm{CI}$ and $\mathrm{C} 2$ are acceleration constants and $\Phi 1$ and $\Phi 2$ are uniformly distributed random numbers in $[0,1]$. The term $\overrightarrow{v_{t}}$ is limited to its bounds. If the velocity violates this limit, it is set to its proper limit, $\omega$ is the inertia weight factor and in general, it is set according to the following equation,

$$
\omega=\omega_{\max }-\frac{\omega_{\max }-\omega_{\min }}{T} \cdot \tau
$$

Where $\omega_{\max }$ and $\omega_{\min }$ is maximum and minimum value of the weighting factor respectively. $\mathrm{T}$ is the number. Based on the updated velocities, each particle changes its position according to the following.

$\overrightarrow{x_{i}}(\tau+1)=\overrightarrow{x_{l}}(\tau)+h(\tau) \overrightarrow{v_{l}}(\tau+1)$

${ }^{\text {Where }} h(\tau)=h_{\max }-\frac{\left(h_{\max }-h_{0}\right) \cdot \tau}{T}$.

Where $h_{\max }$ and $h_{0}$ are positive constants.

According to (24) and (37), the populations of particles tend to cluster together with each particle moving in a random direction. The computation of PSO is easy and adds only a slight computation load when it is incorporated into IGA. Furthermore, the flexibility of PSO to control the balance between local and global exploration of the problem space helps to overcome premature convergence of elite strategy in GAs, and also enhances searching ability. The global best individual is shared by the two algorithms, which means the global best individual can be achieved by the hybrid GA and PSO, also it can avoid the premature convergence in PSO.

\section{Constraints Formulation}

The objective of this method is to minimize some objective functions while satisfying a number of constraints. The constraints are formulated as shown below[7].

\subsection{Minimization of Real Power Loss}

The objective in this paper is to minimize the real power loss (Ploss) in transmission lines of a power system [3]. This is mathematically stated as follows [15], [16].

$P_{\text {loss }}=\sum_{k=(i, j)}^{n} g_{k\left(v_{i}^{2}+v_{j}^{2}-2 v_{i} v_{j} \cos \theta_{i j}\right)}$

where $\mathrm{n}$ is the number of transmission lines, $\mathrm{gk}$ is the conductance of branch $\mathrm{k}, \mathrm{Vi}$ and $\mathrm{Vj}$ are voltage magnitude at bus $\mathrm{i}$ and bus $\mathrm{j}$, and $\theta \mathrm{ij}$ is the voltage angle difference between bus $i$ and bus $j$.

\subsection{Minimization of Voltage Deviation}

It is aimed in this objective that minimizing of the Deviations in voltage magnitudes (VD) at load buses. This is mathematically stated as follows,

Minimize $\mathrm{VD}=\sum_{k=1}^{n l}\left|V_{k}-1.0\right|$

Where $\mathrm{nl}$ is the number of load busses and $\mathrm{Vk}$ is the voltage magnitude at bus $\mathrm{k}$.

\subsection{System Constraints}

In the minimization process of objective functions, some problem constraints which one is equality and others are inequality had to be met. Objective functions are subjected to these constraints shown below. 
Load flow equality constraints,

$$
\begin{gathered}
P_{G i}-P_{D i}-V_{i \sum_{j=1}^{n b} V_{j}}\left[\begin{array}{cc}
G_{i j} & \cos \theta_{i j} \\
+B_{i j} & \sin \theta_{i j}
\end{array}\right]=0, i=1,2 \ldots, n b \\
Q_{G i}-Q_{D i} V_{i \sum_{j=1}^{n b} V_{j}}\left[\begin{array}{cc}
G_{i j} & \cos \theta_{i j} \\
+B_{i j} & \sin \theta_{i j}
\end{array}\right]=0, i=1,2 \ldots ., n b
\end{gathered}
$$

where, nb is the number of buses, PG and QG are the real and reactive power of the generator, $\mathrm{PD}$ and $\mathrm{QD}$ are the real and reactive load of the generator, and Gij and Bij are the mutual conductance and susceptance between bus $i$ and bus $j$.

Generator bus voltage (VGi) inequality constraint,

$$
V_{G i}^{\min } \leq V_{G i} \leq V_{G i}^{\max }, i \in n g
$$

Load bus voltage (VLi) inequality constraint,

$$
V_{L i}^{\min } \leq V_{L i} \leq V_{L i}^{\max }, i \in n l
$$

Switchable reactive power compensations (QCi) inequality constraint,

$$
Q_{C i}^{\min } \leq Q_{C i} \leq Q_{C i}^{\max }, i \in n c
$$

Reactive power generation (QGi) inequality constraint,

$$
Q_{G i}^{\min } \leq Q_{G i} \leq Q_{G i}^{\max }, i \in n g
$$

Transmission line flow (SLi) inequality constraint,

$$
S_{L i}^{\min } \leq S_{L i}^{\max }, i \in n l
$$

where, nc and ng are numbers of the switchable reactive power sources and generators.

\section{Control Strategy}

The operation of PV and Wind system depends on different scenerios. If the total energy or current generated by PV and wind is greater than the required energy by the load, in this case, the excess energy is stored in the battery and battetaking into consideration the rygets charged. When the battery SOC(State of charge) reaches the maximum value, the control unit stops the charging process. Whereas if total energy generated by PV and wind is less than the energy required by the load, the energy deficiency is covered by the storage system. In such case controller puts the batteries in discharge condition. If the battery charge decreases to its minimum state of charge, the controller unit disconnects the load through load controllers (LC), which is otherwise known as load shedding or load shifting method. For optimum selection of control strategy both load following and cycle charging can be adopted [4][5][6].

\section{Objective Function}

The objective function is to minimize losses and to maximize the efficiency of DERs for effective energy management.

\section{Simulation}

For the design of this hybrid system model permits four different tabs to the designer in which the necessary data must be entered by the designer according to the requirement of the system. The tabs contain in general data, optimization, control strategies, and result charts. In this paper the system model consists of PV, WG, Batteries and converter shown in fig 1. In normal operation PV and wind turbines feed the load demand. The excess energy from PV and WG is stored in the battery to full capacity. The main importance of introducing energy storage is to import/export energy depending on the situation [2]

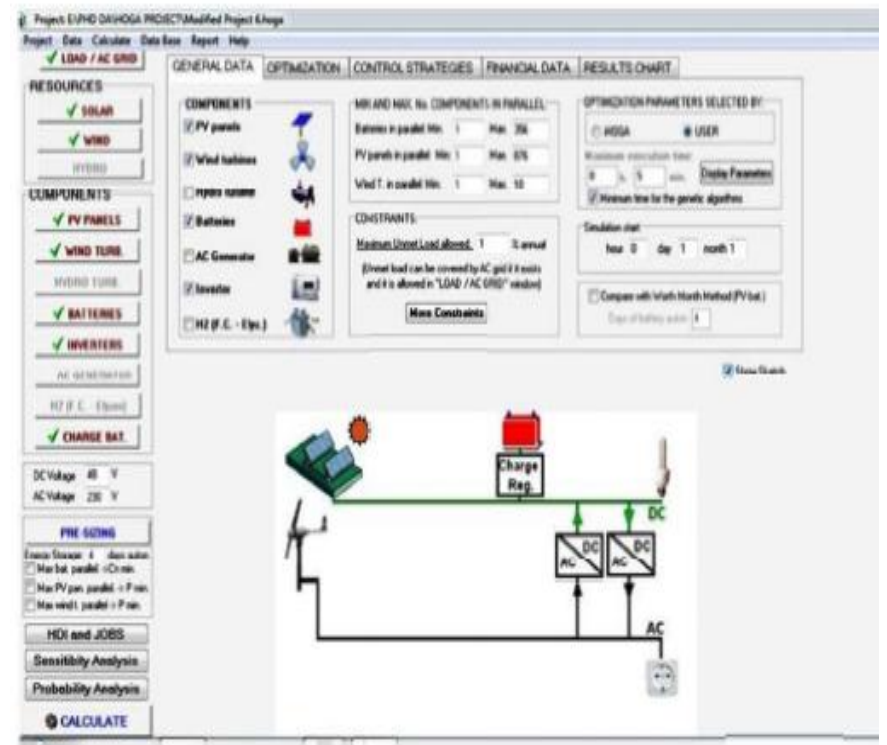

Fig. 1: Hybrid optimizes algorithm simulated Model of PV-Wind system

\section{Results Discussion}

This algorithm performs required objective function. The objective function is to minimize losses, to maximize optimal power flow with efficient energy management methods like hierarchical methods taking into consideration by defining constraints like max load, minimum no of days reserve, nominal battery bank charge, economics of energy cost etc. The hybrid system optimization is studied with sensitivity variables and without sensitivity variables.

\subsection{Optimization without Considering Sensitivity Variables}

The results are shown in fig 2 . The no. of generations evaluated are 15 and total 20504 cases are evaluated for net present cost. The optimum solution for ,PV and Wind energy system, the net present cost is $2756065 \$$ and cost of energy is $0.4 \$ / \mathrm{kwh}$. The solution is optimum as no better solution obtained after 15 generations. The optimum configuration of hybrid $\mathrm{P} \mathrm{V}$-wind renew able energy system consists of PV panels of 10 series x 101 panels parallel with nominal power of $180 \mathrm{Wp}, 24$ batteries series x 42 batteries parallel with the voltage of $12 \mathrm{~V}, 106 \mathrm{Ah}$, Three wind turbines with $72477 \mathrm{~W}$ at $14 \mathrm{~m} / \mathrm{s}$, bidirectional inverter of $150 \mathrm{KVA}$, PV battery charge controller included in bidirectional inverter. 


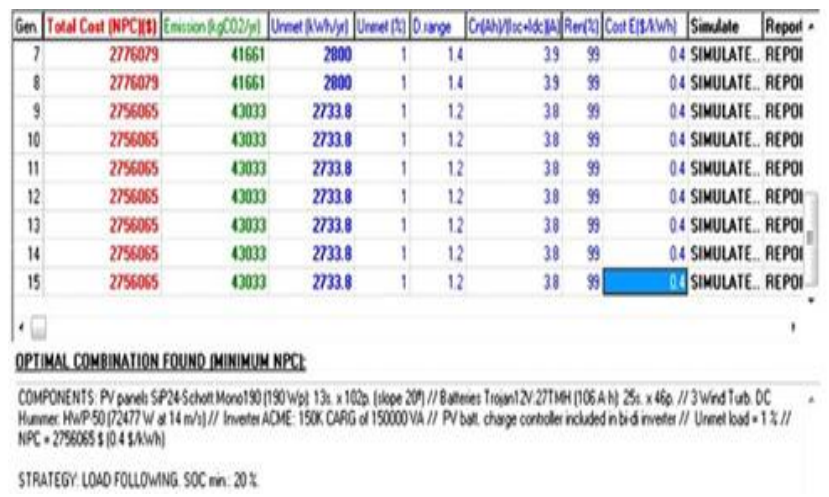

Fig. 2: Optimization results of hybrid PV wind renewableenergy system without sensitivity variable

\subsection{Optimization with Sensitivity Variables}

In this process, first the sensitivity variables are selected as change in global solar radiations and average annual wind speed. Table. 1 shows the results of modified algorithm optimized with sensitivity variables by considering global solar radiations and wind speed. For the different combination of solar radiation and wind speed modified algorithm optimized, the best configuration of hybrid renewable energy system and the results show that for energy combination, system configuration is consisting of PV, wind turbine, battery and converter yields cost of energy of slightly changes from 0.43 to 0.34 $\$ / \mathrm{KWh}$ as shown in table 1 .

Table 1: Optimization Results considering solar panel cost as a sensitivity variables

\begin{tabular}{cccc} 
Sens.\# & Wind Speed $(\mathrm{m} / \mathrm{s})$ & Solar Rad(hW/m2/d) & Lev. cost Energy $(\mathrm{S} / \mathrm{kWh})$ \\
\hline 1 & 3.22 & 5.79 & 0.4 \\
2 & 3.22 & 6.95 & 0.38 \\
3 & 3.22 & 4.05 & 0.42 \\
4 & 3.22 & 5.79 & 0.4 \\
5 & 3.86 & 5.79 & 0.36 \\
6 & 3.86 & 6.95 & 0.35 \\
7 & 3.86 & 4.05 & 0.38 \\
8 & 3.86 & 5.79 & 0.36 \\
9 & 3.7 & 5.79 & 0.37 \\
10 & 3.7 & 6.95 & 0.36 \\
11 & 3.7 & 4.05 & 0.39 \\
12 & 3.7 & 5.79 & 0.37 \\
13 & 4.02 & 5.79 & 0.35 \\
14 & 4.02 & 6.95 & 0.35 \\
15 & 4.02 & 4.05 & 0.37 \\
16 & 4.02 & 5.79 & 0.35 \\
17 & 3.22 & 5.79 & 0.4 \\
18 & 3.22 & 6.95 & 0.38 \\
19 & 3.22 & 4.05 & 0.42 \\
20 & 3.22 & 5.79 & 0.4 \\
\hline
\end{tabular}

\section{Conclusions}

This paper shows optimization of hybrid renewable energy system by considering the effect of sensitivity variables such as global solar radiation, wind speed, and PV panel cost. The results show that for each variable system consisting of solar, wind with battery and converter bring out the most economical and viable solution for the proposed site. The sensitivity analysis helps for proper future predictive planning before installation of the hybrid energy system. By considering the main objective of rural area, planning hybrid renewable energy system has been optimized for economic viability. Economic viability should be the top priority over the technical feasibility while designing the hybrid system for rural electrification in a developing countries like India, where consumers are not always in a position to incur the high costs of power due to their poor economic conditions.

\section{References}

[1] S. Chowdhury, S.P. Chowdhury and P. Crossley,"Microgrids and ActiveDistribution Networks", The Institution of Engineering and Technology, London, United Kingdom, IET Renewable Energy Series 6 , published in the year 2009, available online: www.theiet.org

[2] Ms.JyothiB.Fulzele, Dr. M.B.Daigavane,'Design Optimization of Hybrid PV-Wind Renewable Energy Systems", International Conference on Processing of Materials, Minerals and Energy (July 9th - 30th) 2016, Ongole, Andhra Pradeh, India.., www.m aterialstoday.com /proceedings

[3] K. Lenin, B. Ravindranath Reddy, and M. Surya Kalavathi, "Journal of Industrial and Intelligent Information Vol. 2, No. 3, September 2014.

[4] [12] Anita Gudelj, MajaKrcum, Simulation and optimization of independent renewable energy hybrid system, TOMS transactions on maritimescience, pp. 28-35, 2013.

[5] [13] M. S. Ismail, M. Moghavvemi, T.M.I. Mahlia, Genetic algorithm based optimization on modeling and design of hybrid renewable energysystems, Energy conversion and mangement 85, pp. $120-130$, 2014.

[6] [14] Corsiuc Georgiana, Marza Carmen, C. Emilian, Hybrid solarwind stand alone energy system: a case study, applied mechanics and materals,vol 772, pp. 536-540, 2015.

[7] Document by Ning Kang, Jianhui Wang, Ravindra Singh, and Xiaonan Lu, Center for Energy, Environmental, and Economic Systems AnalysisArgonne National Laboratories, "Interconnection, Integration, and Interactive Impact, Energy Systems Division in January 2017

[8] TomislavDragicevic, Dan Wu, QobadShafiee, and LexuanMeng, "Distributed and Decentralized Control Architectures for ConverterInterfaced Microgrids", in Chinese Journal of Electrical Engineering, Vol.3, No.2, September 2017

[9] Xianyong Feng, Aditya Shekhar, Fang Yang, Robert E. Hebner\&Pavol Bauer, "Comparison of Hierarchical Control and Distributed Control for Microgrid, in Electric Power Components and Systems, Talor and Francis group.

[10] Sandeep Kakran $\square$, SaurabhChanana, "Smart operations of smart grids integrated with distributed generation ",Renewable and Sustainable Energy Reviews, Elsevier, in Dec 2018.

[11] Deloitti Centre for Energy Solutions, "Managing variable and distributed energy resources, Executive summary 2015.

[12] Rumi Rajbongshi, DevashreeBorgohain, SadhanMahapatra "Optimization of PV-biomass-diesel and grid hybrid energy, for rural electrification by using HOMER”, Elsevier, Mar 2017.

[13] Hao Jan Liu, Matthew Backes, Richard Macwan, and Alfonso Valdes, "Coordination of DERs in Microgrids with Cybersecure ResilientDecentralized Secondary Frequency Control ", in Proceedings of the 51st Hawaii International Conference on System sciences 2018

[14] Cesar Hernandez, VíctorGóme, Edwin Rivas,, Universidad Distrital Francisco José de Caldas, Bogotá-Colombia, "Distributed Energetic Resources: Trends, Control and Operation,”, IJET, Nov 20147

[15] Soshinskaya, Mariya; Graus, Wina; Guerrero, Josep M.; Quintero, Juan Carlos Vasquez, "Microgrids: experiences, barriers and success factors", in Renewable and Sustainable Energy Reviews, 2014 Elsevier.

[16] Wenbo Shi, Eun-Kyu Lee, Daoyuan Yao, Rui Huang, Chi-Cheng Chu, and RajitGadh, Smart Grid Energy Research Center, University of California, Los Angeles, USA, "Evaluating Microgrid Management and Control with an Implementable Energy Management System, to appear in IEEE Smart Grid Communications, Venice, Italy, 3-6 Nov. 2014.

[17] Borna\&Khezri, Cogent Mathematics (2015), 2: 1048581 http://dx.doi.org/10.1080/23311835.2015.1048581, Computational Science | Research Article "A combination of genetic algorithm and particle swarm optimization method for solving traveling salesman problem". 